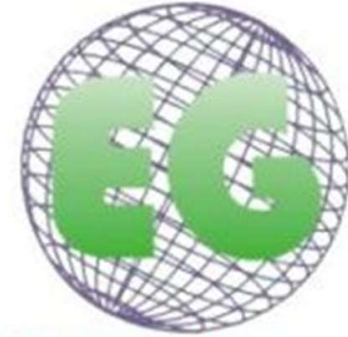

ISSN 1695-6141 N58

\title{
Síndrome de burnout en el Sistema de Salud: el caso de las trabajadoras sociales sanitarias
}

Burnout syndrome in the Health System: The case of social health workers

\author{
Rubén González-Rodríguez ${ }^{1}$ \\ Antonio López-Castedo ${ }^{1}$ \\ Enrique Pastor-Seller ${ }^{2}$ \\ Carmen Verde-Diego ${ }^{1}$
}

${ }^{1}$ Departamento de Análisis e intervención psicosocioeducativa. Universidad de Vigo, Ourense, España. rubgonzalez@uvigo.es

${ }^{2}$ Departamento de Trabajo y Servicios Sociales. Universidad de Murcia. Murcia, España.

https://doi.org/10.6018/eglobal.382631

Recibido: 8/06/2019

Aceptado: 18/09/2019

\section{RESUMEN:}

Objetivo: El objetivo general de esta investigación es medir la prevalencia del síndrome de burnout en el colectivo profesional de trabajadoras sociales sanitarias que ejercen su actividad laboral en los diferentes servicios y centros del Servicio Público de Salud de Galicia (España).

Método: Se ha planteado un diseño de enfoque cuantitativo-analítico y transversal, utilizando como instrumento principal el cuestionario Maslach Burnout Inventory, versión Human Services Suvey. Dicho cuestionario mide tres dimensiones relacionadas con el burnout: Agotamiento emocional, Despersonalización y Realización personal. La recogida de datos se realizó a través de una aplicación de encuestas entre las trabajadoras sociales colegiadas en el Colegio Oficial de Trabajo Social de Galicia.

Resultados: El $86,2 \%$ de la población objeto de estudio son mujeres y un $13,8 \%$ hombres, y sus edades están comprendidas entre los 23 y los 63 años. Los datos de prevalencia del estudio arrojan unos resultados elevados, especialmente en la dimensión de Agotamiento Emocional donde más de la mitad de la población objeto de estudio presenta una puntuación considerada alta.

Conclusión: Al igual que otros colectivos profesionales que ejercen su actividad laboral en el sistema de salud, como es el caso de Enfermería y Medicina, las trabajadoras sociales sanitarias presentan índices elevados de burnout, siendo el Agotamiento Emocional la dimensión más afectada.

Palabras clave: Burnout; Sistemas de salud; Conducta de ayuda; Trabajo Social; Profesionales en salud.

\section{ABSTRACT:}

Objective: The general goal of this research is to measure the prevalence of the burnout syndrome in the professional group of social health workers who perform their work in the diverse services and centers of the Public Health Service of Galicia (Spain).

Method: A quantitative-analytical and cross-sectional design was proposed, using as the main instrument the Maslach Burnout Inventory, Human Services Survey version. This inventory measures three dimensions related to burnout: Emotional Exhaustion, Depersonalization, and Personal 
Fulfillment. Data collection was carried out through a survey among the social workers registered in the Official Association of Social Work of Galicia.

Results: $86.2 \%$ of the population under study are women and $13.8 \%$ are men; their ages range between 23 and 63 years. The study prevalence data yields high scores, especially in the Emotional Exhaustion dimension, where more than half of the population under study obtained a high score.

Conclusion: Like other professional groups that perform their work in the health system, as is the case of Nursing and Medicine, social health workers present high rates of burnout, with Emotional Exhaustion being the most affected dimension.

Key words: Burnout; Health Systems; Helping Behavior; Social Work; Health Occupations.

\section{INTRODUCCIÓN}

El burnout, o agotamiento profesional, es un nuevo fenómeno muy estudiado en las consideradas tradicionalmente como "profesiones de ayuda". Este tipo de profesiones, habitualmente de carácter claramente feminizado, acostumbran estar asociadas a labores de docencia y a la prestación de cuidados. No en vano, la primera referencia al síndrome deriva del análisis realizado por una enfermera en salud mental, que manifestaba presentar un sentimiento de baja moral y distanciamiento de sus pacientes, verbalizando síntomas entre los que destacaba el agotamiento y la desmotivación ${ }^{(1)}$.

Podemos definir el burnout como un tipo de estrés laboral, con etiqueta diagnóstica específica reciente, cuyo elemento esencial es la presencia de desgaste emocional, que suele actuar sobre un estrés laboral previo o simultáneo ${ }^{(2)}$. Se trata, por tanto, de un resultado del estrés de tipo crónico originado por el trabajo y que lleva a enfermar al propio profesional ${ }^{(3)}$.

Maslach y Jackson postularon una tridimensionalidad del síndrome, puesto que consideraron que en él se daban: 1) agotamiento emocional, o pérdida de recursos emocionales para enfrentarse al trabajo; 2) despersonalización, o desarrollo de actitudes negativas, de insensibilidad y cinismo hacia los receptores del servicio prestado; y 3) falta de realización personal en el trabajo, como la tendencia a evaluar el propio trabajo de forma negativa, con baja autoestima profesional, que puede ocurrir entre individuos que trabajan con personas ${ }^{(4)}$.

El Trabajo Social nació vinculado a la ciencia médica a finales del siglo XIX y continúa, a día de hoy, manteniendo una estrecha relación con el ámbito de la salud(5). La presencia de trabajadoras sociales en el campo de la salud, se remonta en España a la década de los cincuenta y sesenta del pasado siglo $X X{ }^{(6)}$. Si bien la mayor parte de este colectivo profesional trabaja en el sistema de servicios sociales, el segundo nicho de empleo donde tiene más representación en ejercicio en España es el sistema de salud ${ }^{(7)}$.

Al igual que otros colectivos profesionales de salud como el de las enfermeras, el trabajo social también es ejercido por una población claramente feminizada. Así, según los últimos datos recabados a nivel estatal, las mujeres suponen el $83 \%$ de las personas colegiadas ${ }^{(8)}$. Asimismo, es necesario destacar que, en España, parece perpetuarse la tradición de que los cuidados informales (no profesionalizados) continúen siendo la opción mayoritaria en nuestra sociedad. La última encuesta sobre discapacidades del Instituto Nacional de Estadística establece que un $70 \%$ de las personas en situación de dependencia son atendidas únicamente con ayuda informal $^{(9)}$. Esto supone que se le dediquen un número de horas elevadas a sus 
cuidados, lo que puede suponer que aumenten las posibilidades de padecer sobrecarga (10), así como de la aparición de problemas físicos o psicológicos que deterioran su salud (11). Por ello, a la vista de estos datos, parece procedente considerar también un análisis sobre estas variables concomitantes, más vinculadas con el ámbito socio-familiar pero que suponen añadir una sobrecarga en atenciones familiares (que no deja de ser un trabajo) a la sobrecarga que, en muchas ocasiones, origina el agotamiento laboral.

Retomando el síndrome de burnout es necesario señalar que las investigaciones sobre el colectivo profesional de trabajadoras sociales son escasas (12). Focalizando ya el análisis en nuestro marco referencial que, como se verá es la Comunidad Autónoma de Galicia, destaca la investigación de Facal-Fondo ${ }^{(13)}$, si bien tiene en consideración solo a profesionales del sistema de servicios sociales. Por ello, nos parece procedente el estudio de este constructo y de sus variables asociadas en el marco de las trabajadoras sociales sanitarias y comparar los datos con otros profesionales de la salud, especialmente con el colectivo de enfermeras.

\section{OBJETIVOS}

\section{Objetivo general}

- Conocer/determinar el grado de sobrecarga en el colectivo profesional de trabajadoras sociales sanitarias del Sistema público de salud de Galicia.

\section{Objetivos específicos}

- Analizar las principales variables sociodemográficas y laborales de las trabajadoras sociales sanitarias del Servicio Gallego de Salud.

- Determinar si el nivel de burnout en este colectivo es diferente al de otros profesionales sanitarios de otras especialidades, especialmente el colectivo de enfermeras, utilizando datos obtenidos en otras investigaciones realizadas en contextos profesionales próximos.

\section{MATERIAL Y MÉTODOS}

Estudio descriptivo, transversal y analítico llevado a cabo entre marzo de 2018 y enero de 2019 en las cuatro provincias de la Comunidad Autónoma de Galicia.

\section{Participantes}

La población objeto de estudio está constituida por las trabajadoras sociales que desempeñan su actividad profesional dentro del Servicio Gallego de Salud (SERGAS). Dado que el cuestionario se distribuyó a través de su entidad colegial de referencia, el único criterio de inclusión definido (además de la pertenencia a la organización) fue que las profesionales estuviesen colegiadas. No se limitó la selección en función del nivel asistencial, por tanto, las participantes desarrollan su labor tanto en atención primaria de salud como en especializada. 
La muestra de análisis resultante fue de 58 sujetos. Estas profesionales del Trabajo Social: 50 mujeres y 8 hombres, con edades comprendidas entre los 23 y los 63 años, trabajan en el Sistema de salud público de la región de Galicia (noroeste de España).

\section{Instrumentos}

Para la recogida de los datos se han empleado dos cuestionarios auto-administrados. Un cuestionario breve, de elaboración propia, donde se han recogido diferentes variables de tipo sociodemográfico, laboral y formativo.

Para evaluar la presencia de síndrome de burnout se utilizó la versión española del cuestionario Maslach Burnout Inventory, versión Human Services Suvey (MBI-HSS), que está orientada a profesionales de la salud ${ }^{(14)}$. El instrumento consta de 22 ítems redactados en forma de afirmaciones y que han de valorarse en una escala tipo Likert. De la mayoría de análisis psicométricos de la escala se desprenden 3 factores, denominados: 1) Agotamiento o cansancio emocional ( $A E)$, con puntuación máxima de 54 puntos; 2) Despersonalización (D), con puntuación máxima de 30 puntos; y 3) Realización personal en el trabajo (RP) con puntuación máxima de 48 puntos.

\section{Procedimiento}

La recogida de datos se realizó a través de una aplicación de encuestas on-line entre las trabajadoras sociales colegiadas en el Colegio Oficial de Trabajo Social de Galicia, organismo a través del cual se publicitó y distribuyó la encuesta. Durante la recogida de la información, así como durante todo el procedimiento de recogida de datos, se mantuvo la voluntariedad en la participación y el anonimato de las respuestas. Todos los cuestionarios recabados se identificaron como completos y no se ha producido mortalidad experimental durante el transcurso de la investigación.

\section{Análisis de los datos}

El análisis de la información se corresponde con resultados de carácter cuantitativo, transversal y descriptivo a partir de análisis de frecuencias y tablas de contingencia. Para su realización se ha utilizado el programa informático SPSS (en su versión 23) y Microsoft Excel.

\section{Tratamiento de los datos}

Previamente a que se realizase el cuestionario se informó a las participantes sobre el anonimato de sus respuestas, presentando su consentimiento informado y respetando lo dispuesto en la Ley Orgánica del 3/2018, de 5 de diciembre, de Protección de datos personales y garantía de los derechos digitales ${ }^{(15)}$.

\section{RESULTADOS}

\section{Características de la muestra}

Las principales características sociodemográficas y profesionales de la población objeto de estudio se presentan en la tabla 1. Como puede observarse, la muestra final resultante la conforman 58 profesionales del Trabajo Social, de los cuales 50 eran mujeres $(86,2 \%)$ y 8 hombres $(13,8 \%)$, superando esta representación la que se 
identificaba a nivel estatal. Sus edades están comprendidas entre los 23 y los 63 años $(\mathrm{Me}=43,9 ; \mathrm{DT}=9,4)$.

Respecto al estado civil, más de la mitad de las participantes está casada o convive en pareja de forma estable $(53,4 \%)$ frente al porcentaje del $32,8 \%$ que se encuentran solteras. Un $62,1 \%$ tiene, al menos, un hijo a su cargo.

Atendiendo a las variables laborales analizadas sorprende el grado de estabilidad contractual de las participantes. Así, más de tres de cada cuatro $(77,6 \%)$ indican disponer de un contrato de tipo fijo. Este dato es congruente con los años de antigüedad en la profesión. De este modo, una de cada tres participantes $(36,2 \%)$ lleva desarrollando su actividad profesional desde hace más de 20 años. El dato también podría apuntar a la escasa renovación de la plantilla estos últimos años.

Ya, por último, si atendemos al nivel de estudios, observamos que más de la mitad de las participantes $(56,9 \%)$ solo disponen de la titulación básica que las habilita para su actividad profesional (título de asistencia social o diplomatura en Trabajo Social). Sin embargo, también puede analizarse desde el enfoque opuesto, esto es, un $43,1 \%$ de las profesionales realizaron un proceso de formación continua, ampliando su titulación inicial con estudios superiores de licenciatura, grado, máster o doctorado.

Tabla 1: Características sociodemográficas y laborales de la muestra

\begin{tabular}{clcc}
\hline Variable & \multicolumn{1}{c}{ Categorías } & Frecuencia & $\%$ \\
\hline \multirow{2}{*}{ Sexo } & Mujer & 50 & 86,2 \\
& Hombre & 8 & 13,8 \\
& Casado/a o en pareja & 31 & 53,4 \\
& Divorciado/a & 6 & 10,3 \\
& Soltero/a & 19 & 32,8 \\
& Viudo/a & 2 & 3,4 \\
& Ninguno & & \\
Número de hijos/as & Uno & 22 & 37,9 \\
& Dos & 15 & 25,9 \\
& Tres & 19 & 32,8 \\
& Más de tres & 1 & 1,7 \\
& & 1 & 1,7 \\
Tipo de contrato & Eventual & 4 & 6,9 \\
& Interino & 9 & 15,5 \\
& Fijo & 45 & 77,6 \\
& & & \\
& Menos de 2 años & 4 & 6,9 \\
& De 2 a 5 años & 5 & 8,6 \\
& De 6 a 10 años & 12 & 20,7 \\
Años de antigüedad en la profesión & De 11 a 15 años & 6 & 10,3 \\
& De 16 a 20 años & 10 & 17,2 \\
& Más de 20 años & 21 & 36,2 \\
& & & \\
& Asistente social & 5 & 8,6 \\
& Diplomatura & 28 & 48,3 \\
& Licenciatura & 5 & 8,6 \\
& Grado & 8 & 13,8
\end{tabular}


Fuente: Datos de la investigación.

\section{Nivel de burnout de las trabajadoras sociales sanitarias}

Existe cierto consenso en que el burnout es una variable continua que se puede manifestar en grado bajo (puntuaciones bajas en $A E$ y $D$, pero altas en RP), moderado (puntuaciones medias en las tres subescalas) o alto (puntuaciones altas en $A E$ y $D$, pero bajas en RP) (16). En base a estas consideraciones pueden estimarse rangos en las puntuaciones, derivados de diferentes estudios e investigaciones. Acostumbran a considerarse los siguientes:

Tabla 2: Estimación del grado, según las puntuaciones en las subescalas

\begin{tabular}{cccc}
\hline & $\begin{array}{c}\text { Agotamiento } \\
\text { emocional (AE) }\end{array}$ & $\begin{array}{c}\text { Despersonalización } \\
\text { (D) }\end{array}$ & $\begin{array}{c}\text { Realización } \\
\text { personal (RP) }\end{array}$ \\
\hline Alto & $\geq 27$ & $\geq 10$ & $\leq 33$ \\
Moderado & $19-26$ & $6-9$ & $34-39$ \\
Bajo & $\leq 18$ & $\leq 5$ & $\geq 40$ \\
\hline
\end{tabular}

Fuente: Datos de la investigación.

Asimismo, también hay estudios que postulan combinar las puntuaciones para obtener una nota de corte a partir de la cual considerar clínicamente el síndrome. De este modo, consideran que existe síndrome de agotamiento profesional si la persona evaluada obtiene algunas de las siguientes puntuaciones: más de 18 puntos en la escala que mide agotamiento emocional; más de 5 puntos en la escala que mide despersonalización; menos de 40 puntos en la escala que mide realización personal en el trabajo ${ }^{(17)}$. Como vemos, los límites para el diagnóstico se corresponden con los recogidos en la tabla 2 , incluyendo el riesgo alto y moderado.

En la tabla 3 se muestran los datos relativos a media, desviación típica, máximos y mínimos de cada una de las escalas teóricas del MBI-HSS para los 58 casos analizados.

Tabla 3: Resultados generales según las subescalas

\begin{tabular}{ccccc}
\hline Escalas & Me & DT & Min & Máx. \\
\hline Agotamiento emocional (AE) & 27,5 & 10,9 & 5 & 47 \\
Despersonalización (D) & 8,3 & 6,4 & 0 & 26 \\
Realización personal (RP) & 34,2 & 6,5 & 20 & 48 \\
\hline
\end{tabular}

Fuente: Datos de la investigación.

En la dimensión $\mathrm{AE}$ la puntuación media para la población objeto de estudio es de 27,5 . Atendiendo al punto de corte, esta puntuación global de la dimensión se situaría en un grado alto. La dimensión $D$ alcanza una puntuación global media de 8,3, dato que sitúa esta dimensión como grado moderado. Esto mismo sucede con la dimensión RP, que alcanza una puntuación global media de 34,2. En todo caso, si analizamos las puntuaciones globales de las tres subescalas, observamos que se verifican los tres indicadores necesarios que postula Grajales para considerar que existe agotamiento emocional ${ }^{(17)}$. 
Tabla 4: Prevalencias en cada una de las subescalas

\begin{tabular}{cccc}
\hline & $\begin{array}{c}\text { Agotamiento } \\
\text { emocional (AE) }\end{array}$ & $\begin{array}{c}\text { Despersonalización } \\
\text { (D) }\end{array}$ & $\begin{array}{c}\text { Realización } \\
\text { personal (RP) }\end{array}$ \\
\hline Alto (\%) & 58,6 & 41,4 & 44,8 \\
Moderado (\%) & 20,7 & 18,9 & 36,2 \\
Bajo (\%) & 20,7 & 39,7 & 19,0 \\
Total & 100 & 100 & 100 \\
\hline
\end{tabular}

Fuente: Datos de la investigación.

Los datos de prevalencia del estudio arrojan unos resultados elevados, especialmente en la dimensión de $A E$ donde más de la mitad de la población objeto de estudio presenta una puntuación considerada alta. Igualmente, en las dimensiones de D y RP, las correspondientes puntuaciones (prevalencia del $41,4 \%$ y $44,8 \%$, respectivamente) sitúan a la población estudiada en unas cifras consideradas de riesgo elevado.

\section{DISCUSIÓN}

Como ya se apuntaba previamente en este estudio, sobre el colectivo profesional de trabajadoras sociales no se han realizado tantas investigaciones sobre burnout como en otras profesiones del ámbito social y de la salud. Más si cabe, centrándose explícitamente en la disciplina del trabajo social sanitario propiamente dicho. Los resultados apuntan a que las trabajadoras sociales que trabajan en el Sistema público de salud presentan índices elevados de burnout, alcanzando puntuaciones consideradas de alto riesgo.

En relación al marco territorial, las puntuaciones de este estudio son más elevadas que las que presentaban en el año 2012 las participantes en una investigación sobre la misma temática, si bien las profesionales eran trabajadoras sociales del Sistema gallego de servicios sociales.

Si atendemos al marco organizacional, las trabajadoras sociales sanitarias presentan puntuaciones elevadas respecto al agotamiento profesional al igual que otros profesionales del Sistema de salud.

Diferentes estudios han constatado niveles altos de burnout entre el colectivo médico (18-20). Los estudios revisados presentan indicadores inferiores al presente estudio. Sorprende, especialmente la diferencia en la dimensión de AE. Así, en los estudios revisados del colectivo médico, las puntuaciones de riesgo alto en esta dimensión se sitúan en un rango entre el $30,6 \%$ y el $43,85 \%$, cuando en nuestra investigación es de un $58,6 \%$.

Otro de los colectivos profesionales perteneciente al Sistema de salud donde más se ha analizado el fenómeno del burnout es el de las enfermeras. Existen múltiples estudios en España realizados en diferentes tipologías de servicios, tales como salud mental, atención primaria u hospitalización ${ }^{(21-24)}$. Sin embargo, es destacable señalar una gran heterogeneidad en los resultados. A este respecto, y dentro del colectivo de enfermeras, se apuntaba que el apoyo social, la resiliencia y las estrategias de afrontamiento de compromiso y de apoyo (adaptativas) constituyen tres herramientas para hacer frente el burnout (25). Por el contrario, el burnout se ha relacionado con altos niveles de estrés ocupacional y con la percepción negativa de la calidad de vida(26). Además, otros estudios evidencian la necesidad de una mayor preocupación 
de los líderes de los servicios del hospital y una concienciación de las enfermeras, que en la mayoría de los casos no se perciben en la enfermedad (27).

Respecto a las trabajadoras sociales se ha evidenciado que, los componentes de empatía pueden prevenir o reducir el burnout y el estrés traumático secundario, por lo que se defiende que la capacitación en habilidades y empatía se incorpore dentro de su formación universitaria ${ }^{(28)}$. De igual modo, al tratarse de una profesión que interviene con frecuencia en problemáticas traumáticas, para evitar el agotamiento debe formarse a los profesionales en el desarrollo de prácticas y estrategias de autocuidado ${ }^{(29)}$.

Como característica diferencial de los colectivos de enfermeras y trabajadoras sociales, señalar la que apuntaban pasadas investigaciones ${ }^{(12,30)}$, y que, a la vista de las puntuaciones, se constata también en este trabajo. En el Trabajo Social, las subescalas de burnout se comportan en modo inverso a otras profesiones como la Enfermería. En general, en el caso de las enfermeras, el burnout se presenta primero como Despersonalización, luego como Agotamiento emocional y, finalmente, con disminución de la Realización personal. Por el contrario, en el caso de las trabajadoras sociales se presenta en primer lugar el Agotamiento emocional, y luego, el reducido logro personal, con una despersonalización de baja ponderación. Como puede observarse, en nuestro estudio es relevante la alta prevalencia en $A E$, sin embargo, a la vista de estas consideraciones, sorprende la prevalencia en la subescala de Despersonalización que no puede considerarse de baja ponderación.

Las altas puntuaciones de nuestra población objeto de estudio, vienen también condicionadas por los años de vida laboral. Recordemos que una de cada tres participantes $(36,2 \%)$ lleva desarrollando su actividad profesional más de 20 años. De igual manera, parece decisivo que, en un colectivo altamente feminizado, existen condicionantes vinculados a los roles normativos de la mujer como prestadora principal de cuidados informales en su contexto familiar. Recordar que un $62,1 \%$ de las participantes tiene, al menos, un hijo a su cargo. En esta investigación no se ha indagado sobre esta cuestión, pero en futuras investigaciones parece necesario preguntar sobre los ascendientes o descendientes (personas mayores o en situación de discapacidad) a los que prestan apoyo y cuidados.

Como limitaciones más significativas se evidencian las dos que se apuntan a continuación. Por un lado, el no poder acceder al total de la población de trabajadoras sociales (tanto colegiadas como no colegiadas). En segundo lugar, sería de interés realizar análisis comparativos de las puntuaciones directas en burnout en otras categorías profesionales que desarrollen su actividad profesional dentro de la misma organización.

Diferentes trabajos de investigación realizados en múltiples países muestran que la atención continuada con personas que exigen altos niveles de implicación emocional genera un importante daño en la salud física y mental de los profesionales ${ }^{(16)}$. Esto evidencia la necesidad de seguir investigando sobre las condiciones laborales de los trabajadores que desempeñan su actividad dentro del Sistema de salud.

Los resultados que se constatan en esta investigación son indicadores relevantes para implementar mejoras dentro de la propia organización. Dichos cambios no deben efectuarse sin tener en consideración los roles normativos de cuidado que las mujeres 
desempeñan tradicionalmente en nuestra sociedad; más si cabe, en colectivos tan altamente feminizados como las enfermeras o las trabajadoras sociales sanitarias.

\section{CONCLUSIONES}

Los profesionales del Trabajo Social que desarrollan su actividad profesional en el Sistema público de salud son mayoritariamente mujeres. Asimismo, de entre las características de su perfil laboral destaca el número de años de antigüedad en la profesión y, por tanto, la falta de incorporación a este sistema de población joven recientemente titulada.

Respecto al nivel de burnout, al igual que en otros colectivos profesionales en los que también se manifiestan relaciones de ayuda, las trabajadoras sociales sanitarias presentan índices elevados en la medición de esta variable. Dichos datos se constatan en esta investigación, donde las participantes presentan puntuaciones incluso más elevadas que otros colectivos profesionales, especialmente en la dimensión de Agotamiento emocional.

\section{Agradecimiento}

Queremos dejar constancia del agradecimiento al Colegio Oficial de Trabajo Social de Galicia, en especial a su Junta de Gobierno, a través del cual se ha conseguido distribuir el cuestionario entre las trabajadoras sociales colegiadas.

\section{REFERENCIAS}

1. Schwartz MS, Will GT. Low morale and mutual withdrawal on a hospital ward. Psychiatry. 1953;16:337-53.

2. Mediano L, Fernández G, Pico V, Arilla JA. EL burnout en los médicos. Gerona: Grupo Ferrer; 2001.

3. Quiceno JM, Alpi SV. Burnout: síndrome de quemarse en el trabajo (SQT). Acta colombiana de Psicología. 2007;10(2):117-25. Disponible en: http://www.redalyc.org/pdf/798/79810212.pdf

4. Maslach C, Jackson S. The measurement of experienced burnout. J Organ Behav. 1981;2:99-113.

5. González-Rodríguez R, Pastor-Seller E, Verde-Diego C. El Trabajo Social en el ámbito sanitario en España. En Pastor-Seller E, Cabello ML, coordinadores. Retos y desafíos del Trabajo Social en el siglo XXI. Una perspectiva internacional comparada México-España. Madrid: Dykinson; 2018. p. 173-84.

6. Ituarte A. Evolución del Trabajo Social en España: historias y perspectivas. Trabajo Social y Salud. 2003;46:69-84.

7. Pastor-Seller E. Situación actual del trabajo social en España: un estudio necesario en España. Revista de Servicios Sociales y Política Social. 2013;103:111-19. Disponible en: http://www.serviciossocialesypoliticasocial.com/situacion-actual-del-trabajosocial-en-espana-un-estudio-necesario-en-espana

8. Consejo General del Trabajo Social. II Informe sobre los servicios sociales en España. Madrid: $\quad$ CGTS; $2015 . \quad$ Disponible https://www.cgtrabajosocial.es/files/5a9ea2319617d/II_ISSE_DIGITAL_2016.pdf

9. Rodríguez A, Rodríguez E, Álvarez, B. Distribución territorial de la dependencia en España y Europa. Papeles Economía Española. 2011;129:27-47. 
10. Rodríguez-González AM, Rodríguez-Míguez E, Duarte-Pérez A, Díaz-Sanisidro E, Barbosa-Álvarez A, Clavería A. Estudio observacional transversal de la sobrecarga de cuidadoras informales y los determinantes relacionados con la atención a las personas dependientes. Aten Primaria. 2017;49(3):156-65. Disponible en: https://www.elsevier.es/es-revista-atencion-primaria-27-articulo-estudio-observacionaltransversal-sobrecarga-cuidadoras-S0212656716301962

11. Cardona D, Segura A, Berberí D, Agudelo M. Prevalencia y factores asociados al síndrome de sobrecarga del cuidador primario de ancianos. Revista Facultad Nacional de Salud Pública. 2013;31(1):30-9. Disponible en: http://www.redalyc.org/articulo.oa?id=12026437003

12. Barría J. Síndrome de burnout en asistentes sociales del servicio nacional de menores de la región metropolitana. Psykhe. 2003;12(1):213-26. Disponible en: http://www.psykhe.cl/index.php/psykhe/article/view/332/312

13. Facal-Fondo T. Prevalencia del síndrome de burnout en trabajadores sociales de los servicios sociales comunitarios. Portularia. 2012;12:59-69. Disponible en: http://www.redalyc.org/pdf/1610/161024645007.pdf

14. Maslach C, Jackson S. Maslach Burnout Inventory Manual. Palo Alto: Consulting Psychology Press; 1986.

15. Jefatura del Estado. Ley Orgánica de Protección de Datos Personales y garantía de los derechos digitales. Ley $3 / 2018$ de 5 de diciembre. Boletín Oficial del Estado, 06.12.2018; 294:119788-857. Disponible en: https://www.boe.es/eli/es/lo/2018/12/05/3

16. Esteras J, Chorot P, Sandín B. El síndrome de burnout en los docentes. Madrid: Pirámide; 2018.

17. Grajales GT. Estudio de validez factorial del Maslach Burnout Inventory. Versión española en una población de profesionales mexicanos. Memorias del CIE. 2001;2:63-82.

18. Caballero MA, Bermejo $F$, Nieto $R$, Caballero F. Prevalencia y factores asociados al burnout en un área de salud. Aten Primaria. 2001;27(5):313-7. Disponible en: https://www.elsevier.es/es-revista-atencion-primaria-27-pdf-S0212656701793730

19. Sos P, Sobrequés J, Segura J, Manzano E, Rodríguez C, García M, et al. Desgaste professional en los medicos de Atención Primaria de Barcelona. Medifam. 2002;12:613-9.

Disponible

en:

http://scielo.isciii.es/scielo.php?script=sci abstract\&pid=S1131-57682002001000002

20. Martínez A, Castillo C, Magaña E, Bru I, Franco A, Segura A. Estudio sobre la prevalencia del burnout en los médicos del Área Sanitaria de Talavera de la Reina. Aten Primaria. 2003;32:343-8. Disponible en: https://www.sciencedirect.com/science/article/pii/S0212656703792944

21. Alba R. Burnout en enfermería: prevalencia y factores relacionados en el medio hospitalario. Rev Cient Soc Esp Enferm Neurol. 2015;41(1):9-14. Disponible en: https://www.elsevier.es/es-revista-revista-cientifica-sociedad-espanola-enfermeria-319articulo-burnout-enfermeria-prevalencia-factores-relacionados-S2013524615000045

22. Torres MD, Sánchez S, Salamanca AB. Enfermera con síndrome de burnout. Revista de enfermería docente. 2015;104:39-43. Disponible en: http://www.indexf.com/edocente/104pdf/10439.pdf

23. Albadalejo $R$, Villanueva $R$, Ortega $P$, Astasio $P$, Calle ME, Domínguez V. Síndrome de burnout en el personal de enfermería de un hospital de Madrid. Rev Esp Salud Publica. 2004;74:505-16. Disponible en: http://scielo.isciii.es/scielo.php?script=sci arttext\&pid=S1135-57272004000400008 24. Sánchez P, Sierra VM. Síndrome de Burnout en el personal de enfermería en UVI. Enferm glob. 2014;13:252-66. Disponible en: http://scielo.isciii.es/scielo.php?script=sci arttext\&pid=S1695-61412014000100012 
25. Arrogante $O$, Aparicio-Zaldivar EG. Herramientas para hacer frente al burnout en el personal de enfermería. Rev Enferm. 2017;40(3):170-7. Accesible en: https://medes.com/publication/120218

26. Vidotti V, Trevisan J, Quina MJ, Perfeito R, Cruz MLC. Síndrome de burnout, estrés laboral y calidad de vida en trabajadores de enfermería. Enferm glob. 2019;55:344-354. Disponible en: http://dx.doi.org/10.6018/eglobal.18.3.325961

27. Santos R, Carvalho FJ, Silva KR, Santos RM, Rodrigues NA, Melo WD. Estrés, burnout y depresión en los profesionales de enfermería de las unidades de cuidados intensivos. Enferm glob. 2019;54:79-93. Disponible en: https://revistas.um.es/eglobal/article/view/337321

28. Wagaman MA, Geiger JM, Shockley C, Segal EA. The Role of Empathy in Burnout, Compassion Satisfaction, and Secondary Traumatic Stress among Social Workers. Soc Work. 2015;60:201-209.

29. Wilson F. Identifying, Preventing, and Addressing Job Burnout and Vicarious Burnout for Social Work Professionals. J Evid Inf Soc Work. 2016;13:479-483.

30. Gil-Monte PR, Pereiró JM. Perspectivas teóricas y modelos interpretativos para el síndrome de quemarse en el trabajo. Anales de Psicología. 1999;15:261-8. Disponible en: https://www.um.es/analesps/v15/v15_2pdf/12v98_05Llag2.PDF 